\title{
ASPECTS DE L'HISTOIRE ENVIRONNEMENTALE COMPARÉE: LA GESTION INTÉGRÉE DE L'EAU (GIRE) DANS LA PERSPECTIVE DE BASSIN VERSANT
}

\section{ASPECTS OF THE COMPARATIVE ENVIRONMENTAL HISTORY: INTEGRATED WATER MANAGEMENT (IWRM) FROM A WATERSHED PERSPECTIVE}

\author{
ELLA HERMON \\ ella.hermon.1@ulaval.ca \\ Université Laval ${ }^{1}$ \\ [RECIBIDO 15/12/2020; ACEPTADO 27/02/2021]
}

\section{RÉSUMÉ}

La grande complémentarité des objectifs et des moyens d'action respectifs des approches modernes de gestion de l'eau s'organise dans une hiérarchie fonctionnelle basée sur un large cadre conceptuel de la gestion intégrée de l'eau (GIRE) en identifiant, d'une part, des buts institutionnelles et sociaux plus prononcés avec la gouvernance et, d'autre part, des visées éthiques avec l'approche de développement durable. Ces approches de gestion ont adopté le bassin versant comme unité de gestion. Pour sa part, l'Antiquité étale des cas comparables pouvant offrir, non seulement de leçons du passé, mais aussi de laboratoire pour enrichir le contenu conceptuel de ces approches de gestion. Ces deux sources du savoir -empirique des Anciens et scientifique des Modernes- évoluent séparément, car la discipline naissante de l'histoire environnementale comparée n'intègre pas un horizon temporel couvrant passé-présent-futur et les études comparées des approches modernes de gestion des ressources en eau ne dépassent pas suffisamment le cadre pragmatique nécessaire pour évaluer des

1 MRSC, professeure émérite, Département d'histoire. Faculté des lettres, Pavillon De Koninck, Bureau 5320, 1030, Avenue des Sciences humaines, Université Laval, Québec (Qc) - GIV A06, Canada.

E. Hermon, "Aspects de l'histoire environnementale comparée: la gestion intégrée de l'eau (GIRE) dans la perspective de bassin versant", RIPARIA 7 (2021), 1-22 
acquis et les défis. Considérées conjointement, les deux visions peuvent générer des pistes de recherche méthodologiques promettantes. Ainsi, nous avons privilégié le système de représentations sociales comme échelle de valeurs qualitative pour évaluer les impacts des interactions société-environnement naturel et identifier l'écosystème des bords de l'eau en tant qu'un système socio-environnemental - Riparia - dont la gestion peut être envisagée à l'échelle hydrographique et topographique du bassin versant.

MOTS-CLES: approches de gestion de l'eau, GIRE, gouvernance, développement durable, représentations sociales, Riparia, bassin versant.

\section{AbStract}

The broad complementarity of the respective objectives and practices of modern approaches to water management is organized in a functional hierarchy based in a comprehensive conceptual framework of integrated water resources management (IWRM) by identifying, on the one hand, more pronounced institutional and social goals with governance and, on the other hand, ethical aims with the sustainable development approach. These management approaches have adopted the watershed as a management unit. For its part, antiquity shows comparable cases that may provide not only lessons of the past, but also a laboratory to enrich the conceptual content of these management approaches. These two sources of knowledge -empirical of the Ancients and scientific of the Moderns- evolve separately, as the nascent discipline of comparative environmental history does not incorporate a time horizon covering past-present-future while comparative studies of modern approaches to water resource management do not go much beyond the pragmatic framework necessary to assess achievements and challenges. Considered jointly, the two approaches may generate promising methodological research avenues. We have chosen therefore the system of social representations as a qualitative value assessment tool for analyzing the impacts of society-natural environment interactions which leads us to identify the waterside ecosystem as a relevant socio-environmental system -Riparia - which may be considered appropriate for the purpose of developing the basin wide management approach.

“Aspects de l'histoire environnementale comparée..." 
KEY WORDS: water management approaches, IWRM, Governance, Sustainable development, Social representations, Riparia, Watershed.

\section{Introduction.}

\section{Dès la définition de la démarche d'analyse des interactions société-environnement naturel aux approches modernes de gestion des ressources en eau}

Nous nous sommes interrogées en 2005 sur l'évolution diachronique des concepts environnementaux modernes afin d'assoir sur des bases théoriques solides la recherche des éléments comparables aux expériences du monde antique, en général, et de l'Empire Romain, en particulier, comme un paradigme interprétatif de la gestion des ressources naturelles dans sa conception et ses pratiques ${ }^{2}$.

Alors que divers courants de pensée tentaient au début du $\mathrm{XX}^{\mathrm{e}}$ de définir et d'élargir le champ sémantique de la notion écologique d'écosystème afin de la rendre compatible avec les études interdisciplinaires, une démarche incontournable, celle des interactions société-environnement ${ }^{3}$, d'une véritable interdépendance entre le monde vivant et son support physique, engendra l'approche écosystémique ${ }^{4}$ qui est une analyse en chaîne de tous les composants d'un écosystème. Sous la contrainte des

\footnotetext{
2 E. Hermon, Les interactions société-environnement naturel; l'évolution diachronique des concepts, in Concepts, pratiques et enjeux environnementaux dans l'empire romain, $\mathrm{R}$. Bedon-E. Hermon (éds), Limoges 2005, 24-26. Nous utilisons ici les définitions que nous avons adoptées et qui figurent dans le glossaire des notions environnementales in E. Hermon, La colonie romaine: espace, territoire, paysage. Les Gromatici entre histoire et droit pour la gestion des ressources naturelles, Besançon 2020, 450-456.

3 Notion variable en fonction de la définition des écosystèmes, soit comme l'équilibre entre société et environnement en fonction de l'intervention anthropique, soit comme la recherche de réciprocité des interactions société-environnement naturel, en considérant l'homme comme partie intégrante de l'écosystème et cette approche sert d'ossature aux concepts environnementaux en rapport avec la gestion de l'eau, E. HERMON, La colonie romaine.., $451 \mathrm{~s}$.

4 Approche de gestion holistique des ressources naturelles en tenant compte de l'ensemble des composantes du milieu, y compris, l'homme qui interviennent dans leur gestion, E. HERMON, La colonie romaine.., 451.
} 
problèmes environnementaux actuels, les praticiens de l'environnement ont mis au service des études environnementales des théories et des méthodes qui ont donné naissance aux approches de gestion intégrée des ressources naturelles en général et de l'eau, en particulier (GIRE) ${ }^{5}$, de gouvernance $^{6}$ et de développement durable ${ }^{7}$. Les changements climatiques, tangibles et prévisibles, que nous vivons ont mis de l'avant l'approche de la GIRE en rapport avec les autres approches mentionnés ci-dessus. Des équipes d'universitaires et des praticiens de l'environnement ont produit une masse critique d'études comparatives de ces approches en vue de proposer des définitions, des méthodologies et des solutions et de consolider ainsi leur contenu conceptuel ${ }^{8}$.

5 Cette approche moderne de gestion préconise le dialogue entre les preneurs des décisions et les utilisateurs des ressources naturelles pour aboutir à une évaluation holistique des interactions société-environnement naturel E. HERMON, La colonie romaine.., p. 454.

6 Terme relativement récent qui émerge dans les années 1980, il fut défini en 1992 par la Banque mondiale en tant que concept directeur, il n'a pas un sens précis et consensuel, mais il implique généralement une éthique de gestion collaboratrice et décentralisée conduisant à l'intervention d'une pluralité d'acteurs. Il s'agit d'un ensemble de règles et des processus collectifs qui engendrent une culture de gestion, https://perspective.usherbrooke.ca

7 Développement qui répond aux besoins du présent sans compromettre la capacité des générations futures de répondre aux leurs. Cette notion est énoncée pour la première fois en 1980 par l'union internationale pour la conservation de la nature (UICN, 1980). Par l'adoption de la loi sur le développement durable, le Québec ajoute à cette définition l'énoncé suivant: le développement durable s'appuie sur une vision à long terme qui prend en compte le caractère indissociable des dimensions environnementale, sociale et économique des activités de développement, assurer, en d'autres mots l'intégrité environnementale à long terme, l'équité sociale et l'efficacité économique, www.ic.gc.ca, consulté le 30 1. 2021.

8 À titre d'exemple, nous considérons ici les études suivantes qui nous semblent représentatives des enquêtes collectives des universitaires et praticiens de l'environnement qui ont analysés les recherches multidisciplinaires sur ces questions afin de dégager l'évolution des approches et de leur contenu conceptuel, de leur méthodologie et dégager des orientations de l'avenir: G. ÖZEROL ET AL., Comparative studies of wáter governance: a systematic review, Ecology and Society 23/4, 2018 https://www.ecologyandsociety.org/vol23/iss4/art43//; J. BUTTERWORTH ET AL., Finding practical approaches to Integrated Water Resources Management, $W$ ater Alternatives 3/1, 2010, 68-81, www.water-alternatives.org; F. MOLLE - PH. Wester, River Basin Trajectories: an Inquiry into Changing Waterscapes, in River Basin

“Aspects de l'histoire environnementale comparée..." 
De telles recherches alimentent la base de données des rapports périodiques du Groupe d'Experts Intergouvernemental sur l'Évolution du Climat (GIEC) crée en 1988 et qui regroupe des représentants de 195 pays. Mais ces études de plus en plus nombreuses peuvent faire elles-mêmes l'objet d'étude sur l'échelle de présent-futur, lorsque l'espace et la durée se mêlent en proportion variable dans les études de cas. Toutefois, un anneau de la chaîne temporelle, celui du passé manque, car il est relégué à l'histoire de l'environnement.

Parallèlement, la volonté de réécrire l'histoire de l'environnement' se fait de plus en plus sentir, car les historiens en quête de nouveaux concepts pour l'analyse des situations historiques, se sont vite aperçus que des problèmes environnementaux comparables se posaient aussi bien aux Anciens qu'aux Modernes, que le recul historique offre un large spectre d'analyse, que les savoirs du passé sont le fondement de l'héritage culturel de gestion des ressources naturelles. Toutes ces directions convergent pour stimuler l'écriture de l'histoire environnementale comparée ${ }^{10}$ qui prenant en ligne de compte,

Trajectories: Societies, Environments and Development, F. MOLLE - PH. WESTER (eds.), C CAB International, Cambridge 2009, 1-19.

${ }^{9}$ L'histoire de l'environnement envisage l'évolution de l'environnement naturel dans ses interactions avec la société, initialement par une approche anthropocentriste, connue en Europe depuis l'École des Annales, et ensuite grâce au courant de la « Nouvelle histoire environnementale » développée en Amérique du Nord vers les années 1970, a fin d'examiner ces rapports en fonction de l'approche écosystémique dans le but de cerner la réciprocité des rapports société-environnement naturel, notamment dans la gestion des ressources naturelles. En revanche, ce courant historiographique a la tendance de situer les origines des problèmes écologiques actuels dans le contexte du passé. Cependant et en reconnaissant l'universalité de l'approche des interactions société-environnement, les deux démarches ont tendance à fusionner, sans abandonner pour autant leurs orientations initiales, E. HERMON, La colonie romaine.., 454.

10 Discipline émergente, elle est ouverte aussi bien aux acquis des sciences sociales qu'aux expériences des gestionnaires de l'environnement; elle favorise la transdisciplinarité dans le développement des concepts environnementaux et ouvre la voie à l'intégration à part entière de l'histoire de la gestion des ressources naturelles dans l'Antiquité en vue de tirer, entre autres, des leçons positives et négatives des expériences du passé en matière de gestion des ressources naturelles, E. HERMON, La colonie romaine.., 454. 
non seulement des données quantifiables et vérifiables d'un passé relativement récent (le XVIII ${ }^{\mathrm{e}}$ siècle), mais également l'étude de cas paradigmatiques du monde antique comme leçon du passé pour le présent et le futur. À notre sens, cette échelle temporelle - passé, présent, futur - conduit à envisager l'histoire environnementale comparée depuis l'Antiquité comme un laboratoire pour la structuration du contenu conceptuel des approches de gestion modernes, en reconstituant les données comparables de l'Antiquité et en offrant ainsi des enseignements sur la pérennité des formes de gestion. Les acquis et les défis des études comparatives des approches modernes de gestion trouve ainsi leur place dans la recherche des axes de recherche dans le cadre de l'histoire environnementale comparée à large échelle chronologique.

Cette confrontation frontale entre passé - présent - futur par le biais de concepts environnementaux dans le cadre de l'histoire environnementale comparée reste à développer. Nous l'avons tenté dans le cadre des travaux de la Chaire de recherche senior du Canada en interaction société-environnement naturel dans l'Empire romain à l'Université Laval et nous la poursuivons dans le cadre des recherches du séminaire Augustín de Horosco à l'Université de Cadiz.

Face à la prolifération des études comparatives consacrées aux différentes approches de gestion des ressources en eau, il convient maintenant d'y dégager des orientations de recherche qui puissent être intégrées à la discipline naissante de l'histoire environnementale comparée et d'ouvrir ce créneau pour l'élaboration de nouvelles méthodologies de recherche afin de cerner l'origine et l'évolution du contenu conceptuel de ces approches de gestion.

Après avoir passé rapidement en revue des acquis des études comparatives des recherches multidisciplinaires des chercheurs et praticiens de l'environnement sur la GIRE, la gouvernance et le développement durable (\& II), nous

“Aspects de l'histoire environnementale comparée..." 
envisageons de nouvelles avenues conceptuelles et méthodologiques en fonction des expériences du monde antique, dans l'esprit de l'histoire environnementale comparée (\& III): Nous esquiserons, en guise de conclusion, quelques orientations posibles d'un dialogue.

\section{Approches comparatives de gestion des ressources en eau}

L'eau en tant que bien public avec sa multiple valeur, sociale, économique et écologique, a guidé l'adoption des principes de sa gestion lors de la conférence des Nations Unies en 1992 sur l'environnement et développement, Earth Summit, Rio de Janeiro, 1992 (ICWE 1992). On a identifié alors des principes tridimensionnels correspondant aux défis de la gestion intégrée des ressources en eau, du sol et des ressources associés (GIRE): mettre l'accent sur l'efficacité économique, sur l'équité d'accès à l'eau et sur la conservation environnementale ${ }^{11}$. Ces trois objectifs consacrent la démarche des interactions société-environnement naturel pour la GIRE. Parallèlement, les autres approches de gestion des ressources en eau, ont été conçues dans le même esprit, en ajoutant ainsi de nouvelles perspectives pour la GIRE, aussi bien au niveau sociétal (la gouvernance) qu'environnemental (développement durable), avec une forte dimension d'éthique de gestion. Si la GIRE assure les modalités de la gestion des ressources en eau, du sol et des ressources associées, la gouvernance assure sa fonction sociale, tout en étant une clé de lecture pour réaliser la durabilité à long terme de la programmation de la gestion en vue d'obtenir les « services de l'eau ». En fait, l'évolution de ces trois approches est complémentaire et elle fait émerger au fur et à mesure de leur application le concept de bassin versant comme unité de gestion de l'espace couvert par la terre et les ressources hydrographiques qu'elle contient, et synthétise les objectifs d'intégration, de

11 F. MolLE, Water for Food - Water for Life: A Comprehensive Assessement of Water Management in Agriculture, Earthscan 2007, 607. 
coopération et de programmation qui caractérisent les différentes approches de gestion ${ }^{12}$.

\subsection{Acquis et défis des synthèses comparatives des approches de gestion de l'eau relies de la GIRE}

L'étude comparative des approches de gestion de l'eau est le défi du début du deuxième millénaire pour les structurer davantage et mettre au point de nouvelles méthodologies, afin de faire face aux problèmes actuels de gestion des ressources en eau et de prévoir l'avenir.

La GIRE a l'avantage de bénéficier d'un cadre conceptuel qui conduit à des avancées méthodologiques et conceptuelles à de différents niveaux, mais le concept est trop générique pour la réalisation simultanée de ces trois objectifs - économique, sociétal et environnemental -, ainsi que pour la coordination intersectorielle et la proposition des pratiques générales valables. En revanche, la gouvernance devient le concept-clé pour la gestion de l'eau en condition des changements climatiques, afin d'élargir le cadre de la prise de décision de la société civile. La comparaison de différentes études a révélé l'utilisation de trois modèles différents: 1) construire une banque de données concrètes et abstraites; 2) utiliser des concepts-cadre des autres disciplines ainsi que leurs expériences afin de combler les vides et d'opérer les transitions; 3) étudier de cas-type pour les problèmes de base sans définir pour autant les critères de sélection. Enfin les objectifs de la GIRE et de la gouvernance se retrouvent dans la promotion de l'éthique de gestion promue par le concept de développement durable et qui consistent: 1) à envisager de maintenir l'intégrité de l'environnement et des écosystèmes; 2) à assurer l'équité sociale et 3) à viser l'efficacité économique.

\footnotetext{
12 F. MoLLE, Water for Food..., 585-625. Le bassin versant est maintenant accepté comme unité de mesure de la gouvernance, bien que les conséquences de la GIRE peuvent avoir également lieu en dehors de ce cadre géophysique et comme ailleurs les solutions sont locales, F. MOLLE - PH. Wester, River Basin Trajectories..., 1-19. Ce dernier livre analyse « la trajectoire » du bassin versant des onze fleuves.
}

“Aspects de l'histoire environnementale comparée..." 
Des enquêtes comparatives des formes d'application des concepts de la GIRE et de la gouvernance ont relevé des conclusions similaires, aussi bien à l'égard de leurs limites que sur la nécessité de proposer des voies alternatives. On a ainsi constaté que leurs valeurs sont facilement transférables d'un concept à l'autre puisqu'on emploie la même démarche d'interactions société-environnement et qu'ils utilisent de différents degrés du processus de prise de décisions pour la gestion de l'eau. Cette dernière restriction dépend, en fait, de la façon de concevoir l'éthique de gestion promue par le concept de développement durable.

La sphère d'application de la GIRE ${ }^{13}$ privilégie notamment l'étude de cas qui présentent des infrastructures développées et des ressources en eau suffisamment abondantes au détriment des régions qui sont en voie de désertification ou des pays en voie de développement. Bien que le bassin versant se profile comme unité de mesure, il y a un grand écart entre les décisions politiques et leur application à ce niveau, tout comme les politiques globales ne rejoignent pas les usagers de l'eau locale qui développent leur propre système de gestion, différent des objectifs de la GIRE, mais conforme aux coutumes locales. En revanche, ces savoirs traditionnels engendrent des systèmes durables et intégrées avec leurs institutions locales qui ont des fonctions holistiques rejoignant les objectifs de l'approche de développement durable. Cette administration locale encourage la coopération entre les acteurs concernés ainsi que l'obtention à l'échelle locale d'un certain l'équilibre de gestion des eaux de surface et de la nappe phréatique. La gestion des unités écologiques comme les milieux ripariens, ces interfaces entre eau et terre, sont souvent plus efficace qu'une prise de décision dans la perspective de bassin versant.

\footnotetext{
13 Voir la bibliographie imposante présentée par J. ButTERWORTH ET AL., Finding practical approaches.., 79-81.
} 
Tout en considérant la GIRE comme une approche de gestion connexe, les comparaisons systématiques des études sur la gouvernance $^{14}$ se réfèrent particulièrement à quelques éléments qui combinent les aspects - institutionnel et social -. En effet, la gouvernance peut inclure, dans un cadre conceptuel plus spécifique, des paramètres pouvant toucher aux lois, aux politiques, à la coopération intersectorielle et à la participation publique. On constate cependant que la plupart de ces études comparatives se basent sur des données primaires quantitatives, sans sélectionner les critères qualitatifs pour les comparaisons. Bien que les pratiques de gouvernance identifiées soient globales en fonction des réformes institutionnelles en termes de bassin versant ou des reformes de la propriété, etc., l'utilisation des frontières juridiques pour la délimitation de cas fait la place au bassin versant comme unité de gestion. Comme pour la GIRE, on met l'accent sur la nécessité d'un choix équilibré de l'importance accordée aux réalités temporelles et spatiales examinées en les interrogeant, par des analyses croisées, sur le respect des critères de justice et d'équité dans la gestion des ressources en eau.

Toutes ces considérations conduisent à concevoir des stratégies alternatives de gestion qui peuvent également recouper les trois approches de gestion des ressources en eau. Dans le cadre de la GIRE, il s'agit de mobiliser les capacités de participation et de coopération de chaque secteur concerné, en adaptant les pratiques pertinentes par une approche holistique, en favorisant des études variées, ainsi que d'élargir le spectre géographique dans le temps et l'espace. L'efficacité des recherches sur la gouvernance devraient se mesurer à deux échelles: par l'administration effective selon les besoins des utilisateurs/usagers à prendre en compte dans le processus décisionnel et de régularisation, ainsi que par la perspective de

\footnotetext{
14 L'étude comparative sur la gouvernance de G. ÖzEROL ET AL., Comparative studies of..., soumet à l'examen 214 publications.
}

“Aspects de l'histoire environnementale comparée..." 
gestion par bassin versant à long terme pour prendre en compte le caractère indissociable des dimensions environnementales, économiques et sociales des activités de développement. Il s'établit, à notre sens, une hiérarchie fonctionnelle entre les trois approches de gestion des ressources en eau - GIRE, gouvernance, développement durable -, mais qui adoptent le bassin versant comme unité de gestión, tout en surpassant sa nature hydrographique et topographique, le bassin versant franchit parfois les frontières institutionnelles de la gestion des ressources en eau ${ }^{15}$. En fin de compte, chacune de ces approches a comme fond commun la démarche des interactions sociétéenvironnement et la dimension conceptuelle de la GIRE qui laisse la place à intégrer les divers aspects développés par les autres approches complémentaires pour la gestion des ressources en eau.

Aux études comparatives de ces approches modernes de gestion, il convient d'incorporer l'histoire environnementale comparée en intégrant ainsi l'Antiquité comme part entière de l'histoire environnementale, car elle présente des traits comparables aux approches modernes de gestion de l'eau. D'ailleurs, le monde romain a l'avantage de présenter les deux perspectives - locale et générale - nécessaires pour l'examen de la GIRE: l'une pragmatique et l'autre normative et nous œuvrons dans ce sens pour enrichir les recherches comparatives sur les approches modernes de gestion de l'eau avec les leçons du passé. Ce chassé-croisé d'influences conduira en fin de compte à renforces les aspects conceptuels de ces approches de gestion.

\section{Les représentations sociales: une interface entre passé, présent et futur}

Notre démarche a un double objectif: s'inspirer des approches modernes de gestion, de leur définition qui revêt des aspects conceptuels, de leur champ d'application et leurs

15 F. Molle, Ph. Wester, River Basin Trajectories... 
méthodologies de recherche pour identifier des équivalents antiques qui pourront éclairer d'un jour nouveau, à un certain degré, des phénomènes semblables aux situations actuelles. Des pistes de recherche pourraient ainsi être suggérées dans cette quête comparative de l'évolution conceptuelle de la gestion des ressources naturelles. De ce fait, et compte tenu de la nature de nos informations, nous avons privilégié le système de représentations sociales comme interface entre les deux composants majeurs de la réalité étudiée - social et environnemental - en interaction. Le système des représentations sociales s'est avéré une échelle de valeurs qualitatives plutôt que quantitatives pour évaluer les impacts des interactions sociétéenvironnement naturel dans le cadre de la gestion intégrée de l'eau où se rencontrent, par une vision instantanée même si limitée, les trois objectifs actuels - économique, social et environnemental - de la gestion intégrée de l'eau qui, selon certaines analyses sont difficilement réalisables simultanément par les approches modernes de gestion, au niveau local aussi bien qu'à un niveau plus large géographiquement. Sous le filtre des perceptions des réalités antiques ressortent, entre autres, les savoirs traditionnels pour une gestion durable, la perception du risque environnemental, des proxy-data sur des variations climatiques au niveau local, mais également une culture juridique au service d'une éthique de la gestion de l'eau ainsi que les transferts culturels. En effet, c'est à travers les représentations sociales que les sociétés antiques transfèrent leurs héritages culturels, tangibles et intangibles, matériels ou écrits, en matière de gestion intégrée de l'eau. C'est à travers les représentations sociales que les savoirs traditionnels conduisent à la perception de l'eau comme patrimoine naturel et culturel $^{16}$ et les pratiques d'adaptation sociale aux intempéries et aux catastrophes de la

16 E. Hermon, Les savoirs traditionnels et la perception de l'eau comme patrimoine naturel et culturel, in L'eau comme patrimoine. De la Méditerranée à l'Amérique du Nord, E. Hermon (ed.), PUL, Québec 2008. coll. « Patrimoine en mouvement », 441-460.

“Aspects de l'histoire environnementale comparée..." 
nature se transforment en résilience ${ }^{17}$, tout en consolidant une véritable éthique de gestion intégrée de l'eau ${ }^{18}$.

\subsection{De l'espace intégré à la gestion intégrée de l'eau}

Bien que la vision œcuménique des Anciens ne soit pas incompatible avec la notion moderne d'écosystème et que les définitions juridiques du ius naturale portent en germe l'approche écosystémique où l'homme fait partie intégrante de l'écosystème ${ }^{19}$, la notion d'écosystème rebute à l'historien du monde antique. En fait, la vision holistique du monde antique ne s'applique pas nécessairement aux interactions entre les composants de l'ensemble de la communauté biotique et son milieu, tel que conçu par la vision écosystémique des Modernes.

C'est la raison pour laquelle nous avons misé sur l'effet rassembleur de la notion d'espace intégré qui donne une identité spécifique au chassé-croisé d'influences entre les différents types d'espace en interaction, où la gestion des ressources naturelles a un effet structurant ${ }^{20}$. En mettant en évidence l'influence de l'héritage romain dans les pratiques modernes de gestion environnementale, nous avons défini la gestion intégrée de l'eau comme un ensemble d'interactions non hiérarchisées entre les différentes espaces intervenant dans la gestion de l'eau et en

\footnotetext{
17 E. Hermon, La gestion intégrée de l'eau entre adaptation et résilience aux variations et changements climatiques dans l'Empire romain, in Riparia - un patrimoine culturel. La gestion des bords de l'eau, E. HeRMON ET A. WATELET (éds.), BAR, International Série 2587, Oxford 2014, 271-286.

18 E. Hermon, Giuristi ed agrimensori per una etica di gestione dell'acqua, in Per una cultura comune dell'acqua dal Mediterraneo all'America del Nord, F.NASTI - F. REDUZZI (eds), Cassino 2012, 71-94.

${ }^{19} \mathrm{La}$ notion d'écosystème et l'approche de gestion écosystémiques trouvent d'ailleurs des bases solides dans la définition des juristes romains du ius naturale, E. Hermon, Mucius Scaevola et le ius naturale - La construction de la résilience dans la gestion du risque d'inondations, JUS 1 (2021), 1-27, à propos des définitions d'Ulpien, Dig. 1.1.1.3, reprise par le Code de Justinien, Inst., I. 2.

20 E. Hermon, Un concept global des bords de l'eau, in Roma, Tevere, litorale. Richerche fra passato e presente, G. CANNOVA ET AL.(curr), Rome 2014, 177-182.
} 
orientetant le processus de négociation entre usagers et preneurs de décisions en matière de gestion intégrée de l'eau (GIRE). ${ }^{21}$

\subsubsection{Riparia: un écosystème spécifique}

Les études comparatives modernes, nous l'avons vu, ont mis en évidence la grande disparité spatiale dans les études de cas actuels, ainsi que l'hétérogénéité des conditions sociétales et environnementales dans les différents contextes examinés. Les recherches écologiques ont identifié comme espace intégré les Riparia (les bords de l'eau), cet espace transitoire entre la terre et l'eau, comme un écosystème propre. Nous l'avons choisi comme objet de recherche pour approfondir les origines épistémologiques de ce terme dans le monde romain. Des enquêtes comparatives des recherches écologiques sur cet écosystème des bords des fleuves ${ }^{22}$, nous avons retenu comme axes de recherche la démarche d'interactions sociétéenvironnement, ainsi que ses paramètres correspondent également à l'ensemble des bords de l'eau - qu'ils soient de nature fluviale, maritime, ou souterraine - en enrichissant ainsi la configuration spatiale du concept de Riparia afin d'en trouver les traits communs des milieux humides en général. Quant à la démarche des interactions société-environnement, nous l'avons saisie en misant sur le système de représentations sociales qui est pour le monde antique le reflet des interactions sociétéenvironnement naturel. Cette approche suggère des éléments de réponse aux limites signalées par les études comparatives des concepts modernes reliés à la gestion des ressources en eau qui ont identifié les milieux humides comme un espace plus pertinent par ses solutions que celui de bassin versant. En effet, les écosystèmes du milieu riparien, particulièrement vulnérables aux risques environnementaux, partagent la conscience de la nécessité de leur conservation et leur adaptation aux transformations du

\footnotetext{
${ }^{21}$ E. Hermon, Représentations sociales .., p. 74

22 R.J. Naiman - H. DéCamps- M.E. McClain, Riparia. Ecology, Conservation and Management of Streamside Communities, Amsterdam-Boston 2005.
}

“Aspects de l'histoire environnementale comparée..." 
milieu. En revanche, la notion de Riparia, qui situe ce milieu au cœur de la dynamique des interactions société-environnement que l'entoure par des cercles concentriques, conçoit à travers les représentations sociales plusieurs dimensions de la gestion du bassin versant (Fig. 1). Cette approche holistique de gestion intégrée des espaces est envisagée par les représentations sociales à trois niveaux - espaces connus, construits et perçus - . C’est « l'espace connu » par la tradition littéraire et la géographie historique qui rend la perspective de bassin versant, entre le temps historique et physique, aux entités hydrographiques qui véhiculent l'eau et charrient les sédiments jusqu'à la mer par un système de pentes et drains naturels ${ }^{23}$, tout en engendrant d'autres formes de milieux humides - marais, delta, lagunes, îles -, sous l'effet combiné des interactions entre la terre, les cours et les plans d'eau. Les pôles d'occupation, colonisation urbaine et rurale, ainsi que des activités économiques, agropastorales, pêches, mines, salines, ports, «construisent » les espaces ripariens avec des pratiques d'aménagement du milieu qui puisent dans les savoirs traditionnels et les transferts culturels afin d'assurer des « services écosystémiques » par la conservation de l'équilibre écosystémique ${ }^{24}$ face à certains effets néfastes de la nature, dont le risque d'inondations. Cette perspective conduit à la troisième vision de l'espace riparien, celle de «l'espace perçu » qui engendre une éthique de gestion pour maintenir à un état stable les fonctions essentielles du système socio-naturel retrouvant dans le bassin versant son unité de gestion.

$* * * * *$

23 J.P. Bravard - F. Petit, Les cours d'eau, dynamique d'un système fluvial, Paris 2000 (1997).

24 O. Petit, Le concept de riparia face aux enjeux contemporains: la nécessité d'une approche interdisciplinaire et intégrée, in Riparia dans l'Empire romain - Pour la définition du concept, E. HERMON (éd.), BAR International Series 2006, Oxford 2010, 13-22. 


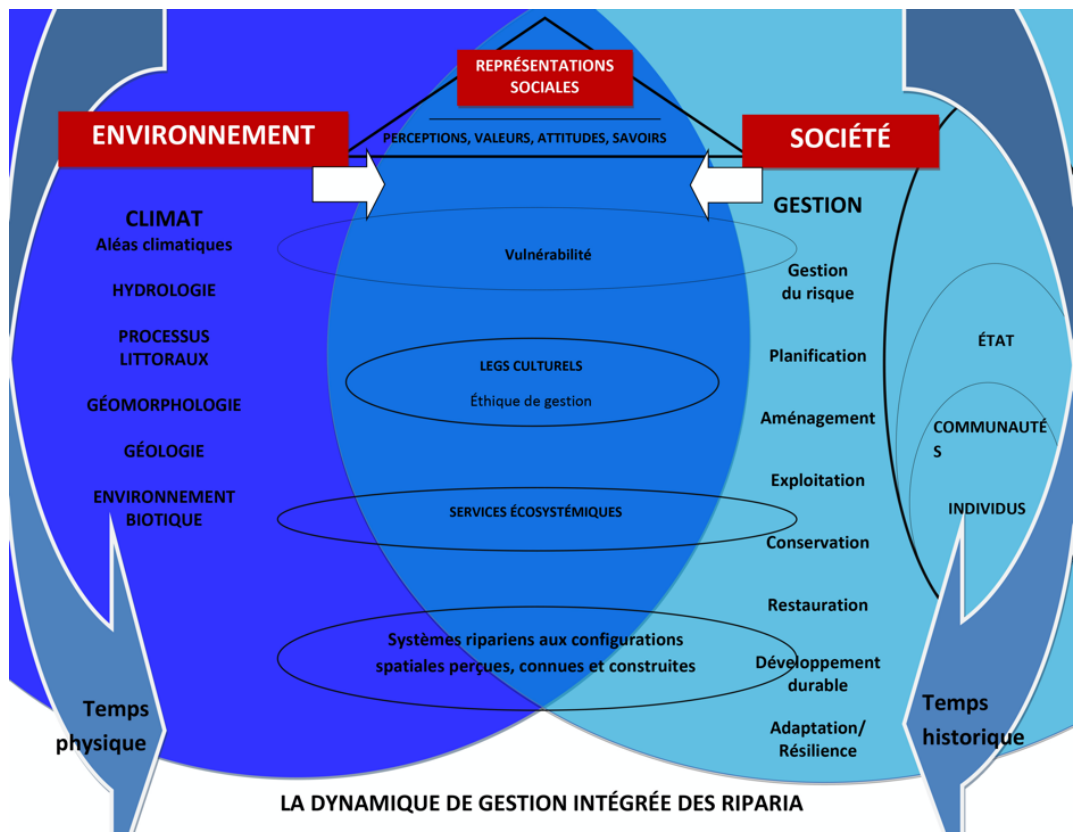

Fig. I. La dynamique de la gestion intégrée des Riparia in E. Heromn-A. WATELET, L'eau comme patrimoine culturel.., 2014, 18.

\subsubsection{Le bassin versant: une échelle hydrographique et topographique}

En l'occurrence, c'est la configuration spatiale du concept de Riparia, qui nous a révélé la perspective de bassin versant en tant qu'échelle hydrographique et topographique des milieux riverains, lacustres, maritimes et humides. Nos recherches sur la gestion de l'eau en interaction avec la terre qui la contient, n'ont identifié que des cas ponctuels, souvent à la merci des interprétations des sources, le plus souvent des représentations sociales qui témoignent néanmoins de la conscience de l'importance du bassin versant et nous limiterons ici à quelques exemples.

La connaissance de la morphologie fluviale a permis aux sources juridiques de délimiter les bords de l'eau, les Riparia qui

“Aspects de l'histoire environnementale comparée..." 
évoluent entre les lits, ordinaire, d'étiage et d'inondation des fleuves $^{25}$ et aux sources techniques des arpenteurs romains de marquer le paysage des milieux humides utilisés pour l'agriculture avec des limites comparables aux marqueurs de la morphologie fluviale $^{26}$. La pratique de l'irrigation par bassin versant des territoires semi-arides est également connue en Espagne romaine. Elle laisse entrevoir sa gestion par des communautés rurales ${ }^{27}$. Des politiques générales d'aménagement paysager au niveau du bassin versant semblent avoir été envisagées pour contrer le risque d'inondation ${ }^{28}$, ainsi que des politiques publiques de fixation périodique du lit d'inondation ${ }^{29}$. Mais ce sont des mesures empiriques et non pas des plateformes de gestion, comme on envisage aujourd'hui pour la planification des politiques par bassin versant. Leur intérêt historique est pourtant indéniable et bien des instances institutionnelles s'en sont inspirées à divers époques. Des projets contemporains de gestion " des espaces de liberté des fleuves " reprennent les préoccupations anciennes à définir les bords de l'eau, les Riparia,

25 P. Jaillette, Le bords de l'eau des fleuves publics au miroir des recueils juridiques de l'Antiquité tardive: textes et traduction, in Riparia dans l'Empire romain, E. HERMON (éd.), BAR International Series 2066, 2006, Oxford 2010, 305-316; L. MAGANZANI, Riparia et phénomènes fluviaux entre histoire, archéologie et droit, in Riparia dans l'Empire romain .., 247-263.

26 J. Peyras, Les Riparia dans les écrits de l'administration civile centrale de l'Antiquité tardive, in Riparia dans l'Empire romain.., 217-230.

${ }^{27}$ F. BELtrán Lloris, La lex rivi Hiberiensis nel suo contesto: i pagi e l'organisazzione dell'irrigazione in Caesar Augusta, in Lex Rivi Hiberiensis. Diritto e technica in una communità di Irrigatione della Spagna Romana, L. MAgAnZAni - C. BuZZACchi (eds.), Napoli 2014, 55-74; E. Hermon, La lex rivi Hiberiensis e la gestione integrata dell'acqua (GIRE) nella Spania romana, in Lex Rivi Hiberiensis ..., 1-32; L.G. LAGÓSTENA BARrIos, Captación y apropiación del aqua para la ciudad. Del paradigma de Roma al caso provincial de la Hispania Ulterior Baetica, in Lex Rivi Hiberiensis.., 33-54.

28 Ph. Leveau, Les inondations du Tibre à Rome: politiques publiques et variations climatiques à l'époque romaine, in, Vers une gestion intégrée de l'eau dans l'Empire romain, E. HERMON (éd.) Rome 2008, 37-146.

29 C. MASI DORIA - C. CASCIONE, Cura riparum, in Riparia dans l'Empire romain..., 283294. 
et considèrent à cet effet le bassin versant ${ }^{30}$. Des anciens pratiques de captage d'eaux des précipitations et de la nappe phréatique en milieux aride sont encore en vigueur de nos jours et les pratiques de stockage et d'irrigation révèlent la gestion communautaire et les rapports ville-campagne.

\section{Conclusion: Le bassin versant: d'une échelle hydrographique à une unité de gestion de la GIRE dans le cadre des Riparia}

Au terme de ce bref aperçu et tout en tenant compte des grands défis posés aujourd'hui par la gestion intégrée de l'eau par bassin versant, on peut s'interroger en quoi le concept de Riparia peut-il servir d'unité écosystémique pour la génération des politiques à l'échelle de bassin versant et comment les expériences du passé peuvent-elles alimenter les réflexions dans ce sens?

Comment les pratiques d'adaptation et de résilience du monde antique, notamment dans la gestion du risque d'inondation, peuvent-elles encore livrer de leçons du passé pour l'application actuelle de la GIRE dans la perspective de bassin versant ? Quelles seraient les formes de coordination en termes de bassin versant des irrigations et des infrastructures hydrauliques monumentales, comme les aqueducs en Espagne romaine $^{31}$ ? Comment le croisement de différentes expressions de représentations sociales du passé antique de protection et de gestion des ressources en eau avec les conflits d'intérêts qu'ils génèrent permet-ils d'hiérarchiser les enjeux, et les visions des acteurs dans une dynamique de gestion intégrée à l'échelle des bassins versants? Alors que des recherches modernes s'interrogent comment mobiliser les représentations sociales pour

\footnotetext{
30 E. Hermon, 'L'espace de liberté de l'eau de Rome à nos jours, Index, 47, 2019, 144 164.

${ }^{31}$ L. G. Lagosténa - CANIZAR J. L. - PONS L. (curr), AQUAM PERDVCENDAM CVRAVTT - Captacion, uso y administración del aqua en las ciudades de la Bética y el Occidente romano, Cadiz 2010.
}

“Aspects de l'histoire environnementale comparée..." 
la gestion des milieux aquatiques par bassin versant, les représentations sociales des Riparia étudiés dans les contextes historiques du passé pourraient sans doute servir d'échantillon de comparaison avec ses constantes et variantes.

En sens contraire, en quoi « les trajectoires » actuelles de bassin versant peuvent-elles inciter la recherche d'éléments équivalents pour la gestion des Riparia dans le monde antique comme les usages concurrents pour l'habitat, l'agriculture, l'industrie, commerce, avec une perception de l'environnement ${ }^{32}$ ? À titre d'exemple, les recherches modernes sur le bassin versant ont démontré que la problématique des «bassins clos » durant les mois secs périodiques ou accidentels génère non seulement de la compétition entre usagers, mais également des certaines formes de gestion, tout en pointant souvent sur des variations climatiques ${ }^{33}$.

Une étude plus systématique des pratiques semblables de la GIRE dans l'espace riparien et à l'échelle du bassin versant dans le monde romain s'avèrerait éclairant pour l'héritage culturel du monde antique.

${ }^{32}$ La question de la continuité écologique a été examinée dans une thèse récente, A. PERRIN, Gouverner les cours d'eau par un concept : étude critique de la continuité écologique des cours d'eau et de ses traductions. Biodiversité et Écologie. Université de Limoges, 2018, Archivesouvertes fr., mais le concept de continuité peut revêtir des formes diverses lorsqu'on considère les représentations sociales du passé antique comme génératrices des politiques de gestion envisagées d'une façon holistique. À titre d'exemple, l'aqua perennis et l'aqua publica, concepts du monde juridique, qui sont des formes de gestion de l'eau et des milieux aquatiques (Riparia), peuvent avoir des implications écologiques à de différents dégrées.

33 F. Molle, Ph. Wester, Ph. Hirsch, River basin closure: Processes, implications and responses. Agricultural water management, 97, 2010, 569-577. www.elsevier.com/locate/agwat/ 


\section{Bibliographie}

F. BELTRÁN Llorís, La lex rivi Hiberiensis nel suo contesto: i pagi e l'organisazzione dell'irrigazione in Caesar Augusta, in Lex Rivi Hiberiensis. Diritto e technica in una communità di Irrigatione della Spagna Romana, L. MAGANZANI-C. BUZZACCHI (eds.), Napoli 2014, 55-74

J. BUTTERWORTH ET AL., Finding practical approaches to Integrated Water Resources Management, Water Alternatives 3(1), 2010, 68-81, www.water-alternatives.org.

C. MASI DORIA - C. CASCIONE, Cura riparum, in Riparia dans l'Empire romain. Pour la définition du concept, in E. HERMON (éd.), BAR International Series 2066, Oxford 2010, 283-294

E. Hermon, Mucius Scaevola et le ius naturale - La construction de la résilience dans la gestion du risque d'inondations, JUS 1 (2021), 1-27

E. HERMON, La colonie romaine: espace, territoire, paysage. Les Gromatici entre histoire et droit pour la gestion des ressources naturelles, Besançon 2020

E. HeRMON, L'espace de liberté de l'eau de Rome à nos jours, Index, 47, 2019, 144-164

E. Hermon, Un concept global des bords de l'eau, in Roma, Tevere, litorale. Richerche fra passato e presente, G. CANNOVA ET AL. (curr), Rome 2014, 177-182

E. HERMON, La lex rivi Hiberiensis e la gestione integrata dell'acqua (GIRE) nella Spania romana, in Lex Rivi Hiberiensis Diritto e technica in una communità di Irrigatione della Spagna Romana, L. MAGANZANI - C. BuZZACCHI (eds.), Napoli 2014, 1-32.

E. HERMON, La gestion intégrée de l'eau entre adaptation et résilience aux variations et changements climatiques dans l'Empire romain, in Riparia - un patrimoine culturel. La gestion des bords de l'eau, E. HERMON ET A. WATELET (éds.), BAR, International Série 2587, Oxford 2014, 271 286

E. HERMON, Giuristi ed agrimensori per una etica di gestione dell'acqua, in Per una cultura comune dell'acqua dal Mediterraneo all'America del Nord, F. NASTI - F. REDUZZI (eds), Cassino 2012, 71-94

E. HERMON, Les savoirs traditionnels et la perception de l'eau comme

“Aspects de l'histoire environnementale comparée..." 
patrimoine naturel et culturel, in L'eau comme patrimoine. De la Méditerranée à l'Amérique du Nord, E. HERMON (éd.), PUL, Québec 2008, 441-460

E. HERMON, Les interactions société-environnement naturel : l'évolution diachronique des concepts, in Concepts, pratiques et enjeux environnementaux dans l'empire romain, R. BEDON - E. HERMON (éds), Limoges 2005, 24-26

P. JAILETTE, Le bords de l'eau des fleuves publics au miroir des recueils juridiques de l'Antiquité tardive: textes et traduction, in Riparia dans l'Empire romain, Pour la définition du concept, in E. HERMON (éd.), BAR International Series 2066, Oxford 2010, 305-316

L. G. LAGÓSTENA BARRIOS, Captación y apropiación del agua para la ciudad. Del paradigma de Roma al caso provincial de la Hispania Ulterior Baetica, in Lex Rivi Hiberiensis Diritto e technica in una communità di Irrigatione della Spagna Romana, L. MAGANZANI - C. BUZZACCHI (eds.), Napoli 2014, 33-54.

L. LAgÓstenA - J.L. CAÑIZAR - L. PONS (cords.), AQUAM PERDVCEND AM CVRAVIT - Captacion, uso y administración del agua en las ciudades de la Bética y el Occidente romano, Cádiz 2010

PH. LEVEAU, Les inondations du Tibre à Rome: politiques publiques et variations climatiques à l'époque romaine, in, Vers une gestion intégrée de l'eau dans l'Empire romain, E. HERMON (ed.), Roma 2008, 37-146

L. MAGANZANI, Riparia et phénomènes fluviaux entre histoire, archéologie et droit, in Riparia dans l'Empire romain. Pour la définition du concept, E. HERMON (éd.), BAR International Series 2066, Oxford 2010, 247-263

F. MOLLE, Water for Food - Water for Life: A Comprehensive Assessement of Water Management in Agriculture, Earthscan 2007

F. MOlLE- PH. Wester, River Basin Trajectories: an Inquiry into Changing Waterscapes, in River Basin Trajectories: Societies, Environments and Development, F. MOLLE - PH. WeSTER (eds.) C CAB International, Cambridge 2009, 1-19

R.J. NAIMAN - H. DÉCAMPs - M.E. MCClain, Riparia. Ecology, Conservation and Management of Streamside Communities, Amsterdam-Boston 2005 
G. ÖZEROL ET AL., Comparative studies of water governance: a syste matic review, Ecology and Society 23/4, 2018

https://www.ecologyandsociety.org/vol23/iss4/art43//

J. Peyras, Les Riparia dans les écrits de l'administration civile centrale de l'Antiquité tardive, in Riparia dans l'Empire romain. Pour la définition du concept, E. HERMON (éd.), BAR International Series 2066, Oxford 2010, 217-230.

A. RIVIÈRE-HONEGGER, M. COTTET, B. MORANDI, dir., Connaître les perceptions et les représentations : quels apports pour la gestion des milieux aquatiques? 